\title{
CONDITIONAL SOLVABILITY OF THE BOUNDARY VALUE PROBLEM OF A SELF-ADJOINT OPERATOR-DIFFERENTIAL EQUATIONS IN A SOBOLEV-TYPE SPACE
}

\author{
Mohamed A. Labeeb ${ }^{1}$, Abdel Baset I. Ahmed ${ }^{\S}$, Labib Rashed ${ }^{3}$ \\ ${ }^{1}$ Egyptian Russian University, EGYPT \\ ${ }^{2}$ Helwan University, EGYPT \\ ${ }^{3}$ Ain Shams University, EGYPT
}

\begin{abstract}
In this paper, in the space of Sobolev type $W_{2}^{5}(R ; H)$ obtained the sufficient conditions of regular solvability of initial-boundary value problem of fifth order operator-differential equations with complicated characteristics on the real axis, these conditions depend only on the operator coefficients of the considered equation. The exact values of norms of the intermediate derivatives operators of the essential part of the investigated equation are obtained.
\end{abstract}

AMS Subject Classification: 34A12, 34G10, 34k10, 35J40, 47D03

Key Words: initial-boundary value problems, operator-differential equation, complicated characteristic, self-adjoint operator, intermediate derivative operator

\section{Introduction}

The theory of initial-boundary value problems of operator- differential equations in Banach or Hilbert space is useful because it offers the possibility of looking at ordinary as well as partial differential operators (see [1]). It should be noted that the essential part of the investigated equation (1.1) has complicated characteristics, thus, in this paper, the investigated equations are of interest, for instance, they arise in modeling the dynamics problems of arches and rings (see [2]). The solvability of initial boundary value problems for higher

Received: January 28, 2019

(c) 2019 Academic Publications

${ }^{\S}$ Correspondence author 
order operator differential equations has been researched by many authors. For example, Aydin A. Gasymov, Araz R. Aliev, V.I. Gorbachuk, M.L. Gorbachuk, Yakubov S. Ya, V.N. Pilipchuk and their followers.

We shall study the following initial-boundary value Problem In a separable Hilbert space $H$

$$
\begin{gathered}
\prod_{k=1}^{5}\left(\frac{d}{d x}-\mu_{k} A\right) u(x)+\sum_{j=1}^{4} A_{j} \frac{d^{5-j}}{d x^{5-j}} u(x)=f(x), \\
x \in R=(-\infty,+\infty), \\
\frac{d^{s} u(0)}{d x^{s}}=0, \quad s=0,1,2,3,
\end{gathered}
$$

where, $A$ is a self-adjoint positively defined operator; $\mu_{1}=1, \mu_{2}=\mu_{3}=\mu_{4}=$ $\mu_{5}=-1$ and $A_{j} ; j=1,2,3,4$ are linear unbounded operators. From now on, the derivatives are accepted according to the distributions theory, [1]. We define the following subspaces.

We consider $f(x) \in L_{2}(R ; H)$, and $u(x) \in W_{2}^{5}(R ; H)$, where

$$
\begin{gathered}
L_{2}(R ; H)=\left\{f(x):\|f(x)\|_{L_{2}(R ; H)}=\left(\int_{-\infty}^{+\infty}\|f(x)\|_{H}^{2} d x\right)^{\frac{1}{2}}<+\infty\right\}, \\
W_{2}^{5}(R ; H)=\left\{u(t): \frac{d^{5} u(x)}{d x^{5}} \in L_{2}(R ; H), A^{5} u(x) \in L_{2}(R ; H)\right\},
\end{gathered}
$$

with the norm (see [1]-[5])

$$
\|u\|_{W_{2}^{5}(R ; H)}=\left(\left\|\frac{d^{5} u}{d x^{5}}\right\|_{L_{2}(R ; H)}^{2}+\left\|A^{5} u\right\|_{L_{2}(R ; H)}^{2}\right)^{\frac{1}{2}} .
$$

Definition 1.1. If for any $f(x) \in L_{2}(R ; H)$ there exists a vector function $u(x) \in W_{2}^{5}(R ; H)$ that satisfies (1.1) almost everywhere in $R$, then it is known as a regular solution of (1.1).

Definition 1.2. If for any function $f(x) \in L_{2}(R ; H)$ there exists a regular solution $u(x) \in W_{2}^{5}(R ; H)$ of (1.1) satisfying the initial boundary conditions (1.2) in the sense that 


$$
\lim _{x \rightarrow 0}\left\|A^{\frac{9}{2}-i} \frac{d^{i} u(x)}{d x^{i}}\right\|_{H}=0, \quad i=0,1,2,3,
$$

and the inequality

$$
\|u\|_{W_{2}^{5}(R ; H)} \leq \text { const }\|f\|_{L_{2}(R ; H)},
$$

holds, then problem (1.1), (1.2) will be regularly solvable.

\section{Main results}

From the theorem of intermediate derivatives (see [6], [7]) if $u(x) \in W_{2}^{5}(R ; H)$, then $A^{5-j} \frac{d^{j} u(x)}{d x^{j}} \in L_{2}(R ; H), j=\overline{1,4}$, and the following inequalities:

$$
\left\|A^{5-j} \frac{d^{j} u(x)}{d x^{j}}\right\|_{L_{2}(R ; H)} \leq c_{j}\|u\|_{W_{2}^{5}(R ; H)}, \quad j=1,2,3,4,
$$

hold.

Equation (1.1) has the following operator form:

$Q u(x) \equiv Q_{0} u(x)+Q_{1} u(x)=f(x)$, where

$Q_{0}=\prod_{k=1}^{5}\left(\frac{d}{d x}-\mu_{k} A\right) u(x)$ and $Q_{1}=\sum_{j=1}^{4} A_{s} \frac{d^{5-j}}{d x^{5-j}}$.

The following theorem provides the association between the norms of operators of intermediate derivatives and the solvability conditions of the problem (1.1), (1.2).

Theorem 2.1. The operator $Q_{0}$ isomorphically maps the space $W_{2}^{5}(R ; H)$ onto the space $L_{2}(R ; H)$, moreover, for $f(x) \in L_{2}(R ; H)$ and equation (1.1) has a solution

$$
u(x)=\int_{-\infty}^{+\infty} G(x-s) f(s) d s+u_{0}(x)
$$

where

$$
G(x-s)=\frac{1}{16}\left\{\begin{array}{c}
e^{A(x-s)} A^{-4}, \text { if } x-s>0 \\
\left(E+2 A(x-s)+2 A^{2}(x-s)^{2}+\frac{4}{3} A^{3}(x-s)^{3}\right) \\
\times e^{-A(x-s)} A^{-4}, \text { if } x-s<0
\end{array}\right\}
$$




$$
u_{0}(x)=\frac{-1}{16}\left\{\begin{aligned}
& \left(E+2 A x+2 A^{2} x^{2}+\frac{4}{3} A^{3} x^{3}\right) \\
& \times \int_{-\infty}^{0} e^{-A(x+s)} A^{-4} f(s) d s \\
& +\left(E-2 A s+2 A^{2} s^{2}-\frac{4}{3} A^{3} s^{3}\right) \\
& \times \int_{0}^{+\infty} e^{-A(x-s)} A^{-4} f(s) d s \\
& +2 A x\left(E-2 A s+2 A^{2} s^{2}\right) \int_{0}^{+\infty} e^{-A(x-s)} A^{-4} f(s) d s \\
& +2 A^{2} x^{2}(E-2 A s) \int_{0}^{+\infty} e^{-A(x-s)} A^{-4} f(s) d s \\
& +\frac{4}{3} A^{3} x^{3} \int_{0}^{+\infty} e^{-A(x-s)} A^{-4} f(s) d s .
\end{aligned}\right.
$$

Proof. First, we find Green's function of equation (1.1) using Cauchy integral where, $A_{j}=0, j=1,2,3,4$, and from inequality (2.1), it is simple to prove that $Q_{0}$ which acts from $W_{2}^{5}(R ; H)$ to $L_{2}(R ; H)$ is bounded (see [8]). When applying Fourier transform to the equation $Q_{0} u(x)=f(x)$, we obtain

$$
(i \xi E-A)(i \xi E+A)^{4} \tilde{u}(\xi)=\tilde{f}(\xi)
$$

where $E$-the identity operator and $\tilde{u}(\xi), \tilde{f}(\xi)$ are the Fourier transforms of the functions $u(x), f(x)$, respectively.

Thus the operator pencil $(i \xi E-A)(i \xi E+A)^{4}$ is invertible and moreover,

$$
\tilde{u}(\xi)=(i \xi E-A)^{-1}(i \xi E+A)^{-4} \tilde{f}(\xi) .
$$

Hence,

$$
u(x)=\frac{1}{2 \pi} \int_{-\infty}^{+\infty}(i \xi E-A)^{-1}(i \xi E+A)^{-4} \tilde{f}(\xi) e^{i \zeta t} d \zeta .
$$

Now we show that $u(x) \in W_{2}^{5}(R ; H)$.

Using the Parseval equality and (2.2), we obtain

$$
\begin{aligned}
& \|u\|_{W_{2}^{5}(R ; H)}^{2}=\left\|\frac{d^{5} u}{d x^{5}}\right\|_{L_{2}(R ; H)}^{2}+\left\|A^{5} u\right\|_{L_{2}(R ; H)}^{2} \\
& =\left\|i \zeta^{5} u \tilde{(\xi)}\right\|_{L_{2}(R ; H)}^{2}+\left\|A^{5} u \tilde{(\xi)}\right\|_{L_{2}(R ; H)}^{2} \\
& =\left\|i \zeta^{5}(i \xi E-A)^{-1}(i \xi E+A)^{-4} \tilde{f}(\xi)\right\|_{L_{2}(R ; H)}^{2} \\
& +\left\|A^{5}(i \xi E-A)^{-1}(i \xi E+A)^{-4} \tilde{f}(\xi)\right\|_{L_{2}(R ; H)}^{2}
\end{aligned}
$$




$$
\begin{aligned}
& \leq \sup _{\zeta \in R}\left\|i \zeta^{5}(i \zeta \mathrm{E}-A)^{-1}(i \zeta \mathrm{E}+A)^{-4}\right\|_{H \rightarrow H}^{2}\|\tilde{f}(\zeta)\|_{L_{2}(R ; H)}^{2} \\
& +\sup _{\zeta \in R}\left\|A^{5}(i \zeta \mathrm{E}-A)^{-1}(i \zeta \mathrm{E}+A)^{-4}\right\|_{H \rightarrow H}^{2}\|\tilde{f}(\zeta)\|_{L_{2}(R ; H)}^{2} .
\end{aligned}
$$

From the spectral decomposition of the operator $A(\sigma(A)$-the spectrum of operator $A$ ) for $\zeta \in R$ we have

$$
\begin{gathered}
\left\|i \zeta^{5}(i \zeta \mathrm{E}-A)^{-1}(i \zeta \mathrm{E}+A)^{-4}\right\|_{H \rightarrow H} \\
=\sup _{\sigma \in \sigma(A)}\left|i \zeta^{5}(i \zeta-\sigma)^{-1}(i \zeta+\sigma)^{-4}\right| \\
\leq \sup _{\sigma \in \sigma(A)} \frac{|\zeta|^{5}}{\left(\zeta^{2}+\sigma^{2}\right)^{\frac{5}{2}}} \leq 1, \\
\left\|A^{5}(i \zeta \mathrm{E}-A)^{-1}(i \zeta \mathrm{E}+A)^{-4}\right\|_{H \rightarrow H} \\
=\sup _{\sigma \in \sigma(A)}\left|\sigma^{5}(i \zeta \mathrm{E}-\sigma)^{-1}(i \zeta \mathrm{E}+\sigma)^{-4}\right| \\
\leq \sup _{\sigma \in \sigma(A)} \frac{\sigma^{5}}{\left(\zeta^{2}+\sigma^{2}\right)^{\frac{5}{2}}} \leq 1 .
\end{gathered}
$$

From (2.4) and (2.5) into (2.3) we obtain:

$$
\|u\|_{W_{2}^{5}(R ; H)}^{2} \leq 2\|\tilde{f}(\zeta)\|_{L_{2}(R ; H)}^{2}=2\|f(x)\|_{L_{2}(R ; H)}^{2} .
$$

Hence, $u(x) \in W_{2}^{5}(R ; H)$.

Using the Banach theorem of the inverse operator, then the operator $Q_{0}$ is an isomorphism from $W_{2}^{5}(R ; H)$ to $L_{2}(R ; H)$.

We formulate exact conditions on regular solvability of problem (1.1), (1.2). Expressed only by its operator coefficients, we must estimate the norms of intermediate derivative operators participating in the perturbed part of the given equation. It follows from Theorem 2.1. that the norm $\left\|Q_{0} u\right\|_{L_{2}(R ; H)}$ is equivalent to the norm $\|u\|_{W_{2}^{5}(R ; H)}$ in the space $W_{2}^{5}(R ; H)$. Therefore by the norm $\left\|Q_{0} u\right\|_{L_{2}(R ; H)}$, the theorem on intermediate derivatives is valid as well. 
Theorem 2.2. When the function $u(x) \in W_{2}^{5}(R ; H)$, then it keeps the following inequalities:

$$
\left\|A^{5-j} \frac{d^{j} u(x)}{d x^{j}}\right\|_{L_{2}(R ; H)} \leq b_{j}\left\|Q_{0} u\right\|_{L_{2}(R ; H)}, j=\overline{1,4,}
$$

true, where $b_{1}=b_{4}=\frac{16}{25 \sqrt{5}}, b_{2}=b_{3}=\frac{6 \sqrt{3}}{25 \sqrt{5}} \quad($ see $[9]-[11])$.

Proof. To establish the validity of inequalities (2.6) we take $Q_{0} u(x)=f(x)$ and apply the Fourier transformation as follow

$$
\begin{gathered}
\left\|A^{5-j}(i \zeta)^{j}(i \zeta \mathrm{E}-A)^{-1}(i \zeta \mathrm{E}+A)^{-4} \tilde{f}(\zeta)\right\|_{L_{2}(R ; H)} \\
\leq \sup _{\zeta \in R}\left\|A^{5-j}(i \zeta)^{j}(i \zeta \mathrm{E}-A)^{-1}(i \zeta \mathrm{E}+A)^{-4}\right\|_{H \rightarrow H}\|\tilde{f}(\zeta)\|_{L_{2}(R ; H)}, \\
j=1,2,3,4 .
\end{gathered}
$$

For $\zeta \in R$ we have:

$$
\begin{gathered}
\left\|A^{5-j}(i \zeta)^{j}(i \zeta \mathrm{E}-A)^{-1}(i \zeta \mathrm{E}+A)^{-4}\right\|_{H \rightarrow H} \\
\leq \sup _{\sigma \in \sigma(A)}\left|\sigma^{5-j}(i \zeta)^{j}(i \zeta \mathrm{E}-\sigma)^{-1}(i \zeta \mathrm{E}+\sigma)^{-4}\right| \\
=\sup _{\sigma \in \sigma(A)}\left|\sigma^{-j}(i \zeta)^{j}\left(i \frac{\zeta}{\sigma}-1\right)^{-1}\left(i \frac{\zeta}{\sigma}+1\right)^{-4}\right| \\
\leq \sup _{\eta=\frac{\xi^{2}}{\sigma^{2}} \geq 0} \frac{\eta}{(\eta+1)^{\frac{5}{2}}}=\frac{1}{25 \sqrt{5}} j\left(j_{/ 2}\right)(5-j) \\
j=1,2,3,4 .
\end{gathered}
$$

Using inequalities (2.7), we have

$$
\begin{gathered}
\left\|A^{5-j}(i \zeta)^{j}(i \zeta \mathrm{E}-A)^{-1}(i \zeta \mathrm{E}+A)^{-4} \tilde{f(\zeta)}\right\|_{L_{2}(R ; H)} \\
\leq b_{j} \| \underset{f(\xi)}{\tilde{(\xi} \|_{L_{2}(R ; H)}}, \quad j=1,2,3,4 .
\end{gathered}
$$


Lemma. The operator $Q_{1}$ continuously acts from $W_{2}^{5}(R ; H)$ to $L_{2}(R ; H)$ provided that the operators $A_{j} A^{-j}, j=1,2,3,4$ are bounded in $H$.

Considering the results found till now (see [12]), we get the possibility to establish regular solvability conditions of the problem (1.1), (1.2).

Theorem 2.3. Let $|\kappa|<2 \lambda_{0}\left(A=A^{*} \geq \lambda_{o} E, \lambda_{o}>0\right)$ for any $u(t) \in$ $W_{2}^{5}(R ; H)$, then holds the inequality (see [13])

$$
\alpha(k)\left(\begin{array}{c}
c_{1}(k)\left\|A_{1} A^{-1}\right\|_{H \rightarrow H}+c_{2}(k)\left\|A_{2} A^{-2}\right\|_{H \rightarrow H}+ \\
+c_{3}(k)\left\|A_{3} A^{-3}\right\|_{H \rightarrow H}+c_{4}(k)\left\|A_{4} A^{-4}\right\|_{H \rightarrow H}
\end{array}\right) \prec 1,
$$

where

$$
\begin{aligned}
& c_{1}(k)=\left[1+\frac{4 \lambda_{0}\left|\lambda_{0}+k\right|}{\left(2 \lambda_{0}+k\right)^{2}}\right]^{\frac{3}{2}}, \quad c_{2}(k)=\frac{2 \lambda_{0}}{2 \lambda_{0}+k}\left[1+\frac{4 \lambda_{0}\left|\lambda_{0}+k\right|}{\left(2 \lambda_{0}+k\right)^{2}}\right], \\
& c_{3}(k)=\frac{4 \lambda_{0}^{2}}{\left(2 \lambda_{0}+k\right)^{2}}\left[1+\frac{4 \lambda_{0}\left|\lambda_{0}+k\right|}{\left(2 \lambda_{0}+k\right)^{2}}\right]^{\frac{1}{2}}, \quad c_{4}(k)=\frac{8 \lambda_{0}^{3}}{\left(2 \lambda_{0}+k\right)^{3}},
\end{aligned}
$$

and

$$
\alpha(k)=\left\{\begin{array}{cl}
\frac{\lambda_{0}}{2^{\frac{1}{2}}\left(2 \lambda_{0}^{2}-k^{2}\right)^{\frac{1}{2}}}, & \text { if } 0 \leq \frac{k^{2}}{4 \lambda_{0}^{2}} \prec \frac{1}{3}, \\
\frac{2 \lambda_{0}|k|}{4 \lambda_{0}^{2}-k^{2}}, & \text { if } \frac{1}{3} \leq \frac{k^{2}}{4 \lambda_{0}^{2}} \prec 1 .
\end{array}\right.
$$

Theorem 2.4. Suppose that the operators $A_{j} A^{-j}, j=\overline{1,4}$, are bounded in $H$ and they satisfy the inequality

$$
\sum_{j=1}^{4} C_{j}(k) \alpha(k)\left\|A_{5-j} A^{-(5-j)}\right\|_{H \rightarrow H} \prec 1,
$$

where the numbers $C_{j}(k), j=1,2,3,4$, and $\alpha(k)$ are determined in Theorem 2.3. Then the problem (1.1), (1.2) is regularly solvable.

Proof. $f(x) \in L_{2}(R ; H), u(x) \in W_{2}^{5}(R ; H)$ and by Theorem (1.1), there exist a bounded inverse operator to $Q_{0}$, which acts from $L_{2}(R ; H)$ to $W_{2}^{5}(R ; H)$, then after replacing $Q_{0} u(x)=w(x)$ in equation (1.1), it can be written as $\left(E+Q_{1} Q_{0}^{-1}\right) w(x)=f(x)$.

Now we prove under the theorem conditions (see [13], [14]) that

$$
\left\|Q_{1} Q_{0}^{-1}\right\|_{L_{2}(R ; H) \rightarrow L_{2}(R ; H)}<1 .
$$


By Theorem (2.3) we have:

$$
\begin{aligned}
& \left\|Q_{1} Q_{0}^{-1} w\right\|_{L_{2}(R ; H)}=\left\|Q_{1} u\right\|_{L_{2}(R ; H)} \leq \sum_{j=1}^{4}\left\|A_{j} \frac{d^{5-j} u}{d x^{5-j}}\right\|_{L_{2}(R ; H)} \\
& \leq \sum_{j=1}^{4}\left\|A_{j} A^{-j}\right\|_{H \rightarrow H}\left\|A^{j} \frac{d^{5-j} u}{d x^{5-j}}\right\|_{L_{2}(R ; H)} \\
& \quad \leq \sum_{j=1}^{4} C_{j}(k) \alpha(k)\left\|A_{j} A^{-j}\right\|_{H \rightarrow H}\left\|Q_{0} u\right\|_{L_{2}(R ; H)} \\
& \quad=\sum_{j=1}^{4} C_{j}(k) \alpha(k)\left\|A_{j} A^{-j}\right\|_{H \rightarrow H}\|v\|_{L_{2}(R ; H)} .
\end{aligned}
$$

Consequently,

$$
\left\|Q_{1} Q_{0}^{-1}\right\|_{L_{2}(R ; H) \rightarrow L_{2}(R ; H)} \leq \sum_{j=1}^{4} C_{j}(k) \alpha(k)\left\|A_{j} A^{-j}\right\|_{H \rightarrow H} \prec 1 .
$$

Thus, the operator $E+Q_{1} Q_{0}^{-1}$ is invertible in $L_{2}(R ; H)$, therefore $u(x)$ can be determined by $u(x)=Q_{0}^{-1}\left(E+Q_{1} Q_{0}^{-1}\right)^{-1} f(x)$, and moreover

$$
\begin{gathered}
\|u\|_{W_{2}^{5}(R ; H)} \leq\left\|Q_{0}^{-1}\right\|_{L_{2}(R ; H) \rightarrow W_{2}^{5}(R ; H)} \\
\times\left\|\left(\left(E+Q_{1} Q_{0}^{-1}\right)\right)^{-1}\right\|_{L_{2}(R ; H) \rightarrow L_{2}(R ; H)}\|f\|_{L_{2}(R ; H)} \\
\leq \text { const }\|f\|_{L_{2}(R ; H)} .
\end{gathered}
$$

\section{Conclusion}

In the real axis, for the fifth order self-adjoint differential operator with complicated characteristics, we demonstrated the association between the coefficients of the differential operator and conditions of the regular solvability of problem (1.1)-(1.2). We estimated the norms of intermediate derivative operators which appear in the essential part of the investigated equation. The norms of the linear operators $\left(A_{j}, j=1,2,3,4\right)$ participating in the second part estimated and used to formulate the exact solvability conditions.

\section{References}

[1] E. Hille, R. Phillips, Functional Analysis and Semi-Groups, Moscow (1962), In Russian. 
[2] J.L. Lions and E. Magenes, Non-Homogeneous Boundary Value Problems and Their Applications, Springer-Verlag, Berlin Heidelberg, New York (1972).

[3] V.I. Gorbachuk and M.L. Gorbachuk, Boundary Value Problems for Operator-Ddifferential Equations, Naukova Dumka, Kiev, (1984), In Russian.

[4] S.G. Krein, Linear Differential Equations in a Banach Space, Nauka, Moscow (1967).

[5] S.Ya. Yakubov, Linear Differential Operator Eequations and Their Applications, Elm, Baku (1985), In Russian.

[6] F.S. Lachinova, Solvability of a class of parabolic operator-differential equations of third order, Proc. of IMM of NAS of Azerbaijan, XXXIX (2013), $77-86$.

[7] R.P. Agarwal, M. Bohner, and V.B. Shakhmurov, Maximal regular boundary value problems in Banach-valued weighted space, Boundary Value Problems, 2005, No 1 (2005), 9-42.

[8] A.R. Aliev and A.A. Gasymov, On the correct solvability of the boundaryvalue problem for one class operator-differential equations of the fourth order with complex characteristics, Boundary Value Problems, 2009, Article ID 710386 (2009), 1-20.

[9] A.R. Aliev and A.S. Mohamed, On the well-posed of a boundary value problem for a class of fourth order operator differential equations, Differential Equations, 48, No 4 (2013), 596-598.

[10] A. Baset I. Ahmed and M.A. Labeeb, Solvability of a class of operatordifferential equations of third order with complicated characteristic on the whole real axis, Open Access Library Journal, 5 (2018), 1-5.

[11] A.R. Aliev and A.L. Elbably, Well-posedness of a boundary value problem for a class of third-order operator-differential equations, Boundary Value Problems, 2013 (2013), 1-15.

[12] A.R. Aliev and A.L. Elbably, On the solvability in a weight space of a thirdorder operator-differential equation with multiple characteristic, Doklady Mathematics, 85, No 2 (2012), 233-235. 
[13] A.R. Aliev, On the solvability of a class of operator differential equations of the second order on the real axis, J. of Mathematical Physics, Analysis, Geometry, 2, No 4 (2006), 347-357.

[14] A.R. Aliev, On the solvability of the equations containing in the main part the operators of the form $-\frac{d^{3}}{d t^{3}}+A^{3}$ in the weight space, Transaction of NAS of Azerbaijan (2006), 9-16. 